\title{
Antonio Lillo, Terry Victor. A Dictionary of English Rhyming Slangs. Berlin/Boston: De Gruyter Mouton, 2017, 1358 p.
}

Besprochen von Félix Rodríguez González: Rodríguez González, Departamento de Filología Inglesa, Universidad de Alicante, Ap. 99, 03080 Alicante, España,

E-Mail: frodriguez@ua.es

https://doi.org/10.1515/les-2019-0009

This dictionary is a comprehensive survey of rhyming slangs in the Englishspeaking world and is a milestone in the lexicography of this subset of lexis. Accustomed as I am to reading about the phenomenon of rhyming slang (RS), I was at first surprised at the pluralized slangs of the title. But a closer look made me realize that it could not be more precise in its description on several accounts. First, due to the depth and breadth of the study, for it is not a mere description of the oft-quoted Cockney RS originally spawned in London as a distinct lingo among the underclasses and the underworld. In fact, RS is used among a wide variety of social groups and is not limited to marginal sectors, and the regional dialects represented in this compilation are equally varied and comprise areas spattered over the Anglophone world: the UK, Australia, Ireland, the US, Canada, South Africa, India, New Zealand, Sri Lanka, Zambia, etc.

In addition, this dictionary examines an ample range of variant structural patterns of RS among which certain categories stand out. For instance, "perfect" (the well-known apples and pears 'stairs', or simply apples), "imperfect” (Leslie Crowthers 'trousers'), "compounded" (Barney Rubble 'a laugh', by virtue of the rhyme on bubble, a shortening of bubble bath, with the second element pronounced barf), "covert” (bunny 'talk', on the analogy of rabbit < rabbit and pork 'talk') and "pseudo" (Dick Van Dyke 'a lesbian', punning on the homophone dyke).

Bearing all this in mind, the resulting macrostructure of the dictionary is no surprise. The authors have managed to collect over 9,000 headwords in use as of the 1830s, and in so doing they have outnumbered by six times Julian Franklyn's classic A Dictionary of Rhyming Slang, whose second edition was published in 1961. This monumental tour de force has been patiently compiled over a number of years by the two authors, with no additional aid. The partnership in this linguistic enterprise could not have been more appropriate. Antonio Lillo is a long-standing scholar in the field, as he has dedicated half of his professional life to the domain of rhyming slang, proof of which are his master's thesis, his Ph.D. dissertation, and an extensive list of research papers on slang published in a variety of internationally journals of renown. Lillo's initial training as a lexicographer and a phonetician, plus his fine ear for speech patterns and accents, have 
also helped him significantly in this endeavour. Terry Victor has two equally important assets: his experience as a lexicographer and student of slang (he is coeditor of The New Partridge Dictionary of Slang and Unconventional English, now in its second edition, and his work as an actor, director and broadcaster on language.

The value of this dictionary does not lie only in the extensive compilation as such. One of the most important difficulties in a piece of this nature is, first, identifying and classifying words and phrases as true candidates for "rhyming slang" according to well-defined criteria. Slang is not always easy to pinpoint due to its blurred boundaries. This is reflected in the various labels used in dictionaries of general usage in marking entries: coll(oquial), inf(ormal), fam(iliar), vulg (ar), taboo, etc. "Rhyming slang”, in marked contrast, stands out as a distinct subtype because of rhyme, although the complex structure of the underlying and omitted rhymed words is often revealed a hard task. It is difficult since it requires a wide linguistic knowledge and familiarity with the culture and sociology of the English-speaking world, especially urban Britain.

This dictionary follows the format of other dictionaries based on historical principles, such as the $O E D$. Each entry includes conventional part-of-speech labels supplemented with specific kinds of nouns (such as nicknames and proper nouns). The various uses it contains are ordered chronologically and the dates are marked in boldface to facilitate search. Given the sheer size and scope of the dictionary, the information may seem too condensed to the eye, but the layout and good typographical presentation make reading easy. Another tenet of a good dictionary of lexical usage is the resort to a large number of quotations illustrating terms; they should also furnish date and location of use, give validity to the words' permanence and, ideally, trace their origin and evolution.

On bringing together the wide mass of miscellaneous items listed, Lillo and Victor have needed to explore multifarious and variegated sources (oral examples, broadcasts, and extracts of digital discourse, among many others) as well as citations from printed works. Thus the collected items include those gathered from present-day use plus those recorded before.

However, incorporating published material without further questioning has its own risks, and here the authors have been especially cautious and in several instances have excluded items that proved false elaborations of non-rhyming slang words. They have also spotted some "ghost words", that is, words that have made their way into dictionaries or other works by mistake, and due to various reasons: misprints, misreadings, etymological confusion or unevidenced interpretations.

As a lexicographer I also feel that one of the elements adding value to a dictionary, and warming a lexicographer's heart, is finding uses preceding the 
ones recorded in earlier works. And this dictionary, in effect, contains a significant number of antedatings. One clear example is Hampton Wick 'the penis', rhyming on the synonymous prick and dick dated 1930 in a major slang dictionary, and here antedated to 1898. Equally significant is the postdating of some terms which may pass unnoticed by lexicographers if they previously became obsolete for a long span of time.

The body of A Dictionary of English Rhyming Slangs is preceded by a 22-page introduction which is a gem for dictionary makers and slang researchers, along with lovers of slang in general and RS in particular, because of the array of useful recommendations and insightful remarks. Over the years RS has greatly lost its connotations of secrecy linked to the underworld where it originated, and has gained respectability and become attractive to the general public due to the playful humour and continuous inventiveness displayed by its speakers. The careful focus on this area of lexis that characterizes this book makes it a muchneeded work of reference, since it fills a niche in the study of slang and is a substantial contribution to the field. 